\title{
Predicting Structural Details of the Sodium Channel Pore Basing on Animal Toxin Studies
}

\author{
Denis B. Tikhonov ${ }^{1 *}$ and Boris S. Zhorov ${ }^{1,2}$ \\ ${ }^{1}$ Sechenov Institute of Evolutionary Physiology and Biochemistry, Russian Academy of Sciences, Saint Petersburg, Russia, \\ ${ }^{2}$ Department of Biochemistry and Biomedical Sciences, McMaster University, Hamilton, ON, Canada
}

\section{OPEN ACCESS}

Edited by:

Alexander A. Vassilevski, Institute of Bioorganic Chemistry

(RAS), Russia

Reviewed by:

Vladimir Yarov-Yarovoy, University of California, Davis, United States

Jeremiah Osteen,

Vertex Pharmaceuticals

(United States), United States

${ }^{*}$ Correspondence:

Denis B. Tikhonov

denistikhonov2002@yahoo.com

Specialty section:

This article was submitted to Pharmacology of Ion Channels and Channelopathies,

a section of the journal

Frontiers in Pharmacology

Received: 25 May 2018

Accepted: 20 July 2018

Published: 07 August 2018

Citation:

Tikhonov DB and Zhorov BS (2018) Predicting Structural Details of the Sodium Channel Pore Basing on Animal Toxin Studies.

Front. Pharmacol. 9:880. doi: 10.3389/fphar.2018.00880
Eukaryotic voltage-gated sodium channels play key roles in physiology and are targets for many toxins and medically important drugs. Physiology, pharmacology, and general architecture of the channels has long been the subject of intensive research in academia and industry. In particular, animal toxins such as tetrodotoxin, saxitoxin, and conotoxins have been used as molecular probes of the channel structure. More recently, X-ray structures of potassium and prokaryotic sodium channels allowed elaborating models of the toxin-channel complexes that integrated data from biophysical, electrophysiological, and mutational studies. Atomic level cryo-EM structures of eukaryotic sodium channels, which became available in 2017, show that the selectivity filter structure and other important features of the pore domain have been correctly predicted. This validates further employments of toxins and other small molecules as sensitive probes of fine structural details of ion channels.

Keywords: conotoxins, homology modeling, ligand docking, local anesthetics, tetrodotoxin

\section{INTRODUCTION}

Voltage-gated sodium channels belong to the superfamily of voltage-gated ion channels, which also include calcium, potassium, glutamate-gated, and other channels. Eukaryotic VGSCs comprise $\alpha$ and $\beta$ subunits. The large $\alpha$-subunit, which folds from a single polypeptide chain of four homologous repeats, contains a pore domain and four VSDs domains. Each repeat comprises six transmembrane helical segments (S1-S6) connected by extra- and intracellular loops. Segments S1-S4 form VSDs. Segments S5 (the outer helices), S6 (the inner helices), and extracellular membrane reentering P-loops between S5 and S6 contribute to the pore domain (Figure 1A). The P-loops contain membrane-descending (P1) and membrane-ascending (P2) helices with residues between P1 and P2 contributing to the selectivity filter. In eukaryotic VGSCs, the selectivity filter DEKA ring, which contains $\mathrm{D}, \mathrm{E}, \mathrm{K}$, and A residues, borders the extracellularly exposed outer pore and the inner pore that is exposed to the cytoplasm in the open channel (Figure 1B). The activation gate, which is composed of the cytoplasmic parts of S6s, forms a tight ion-impermeable bundle in the closed state. Upon membrane depolarization the $\mathrm{S} 4$ helices, which contain positively charged residues, shift in the extracellular direction, thus inducing movements of the S4-S5 linker helices and finally the activation gate opening. In the open state, S6s diverge to form a wide inner vestibule.

Abbreviations: $\mu$-CTX, $\mu$-conotoxins; EM, electron microscopy; LRET, luminescence resonance energy transfer; STX, saxitoxin; TTX, tetrodotoxin; VGSCs, voltage-gated sodium channels; VSD, voltage-sensing domain. 


\section{A}

\section{TTX, STX, $\mu-C T X$}

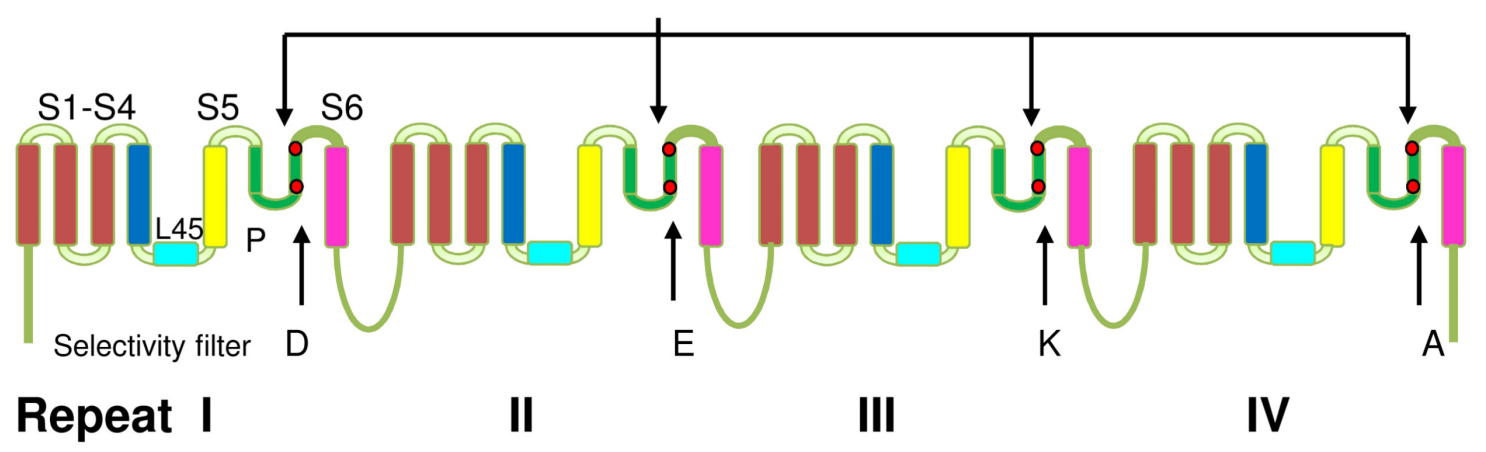

B

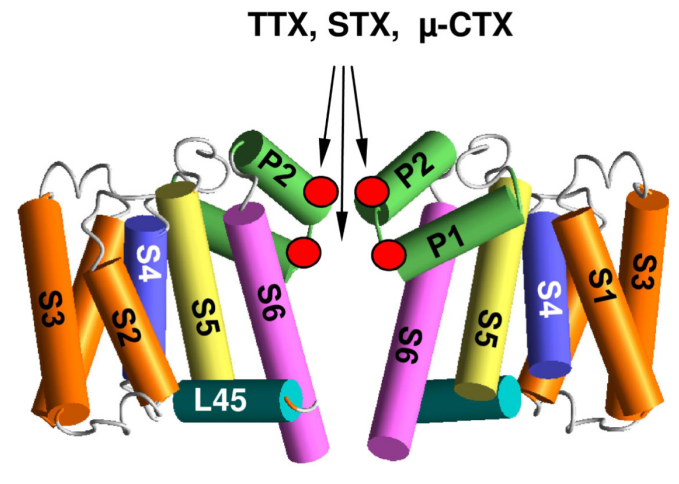

C
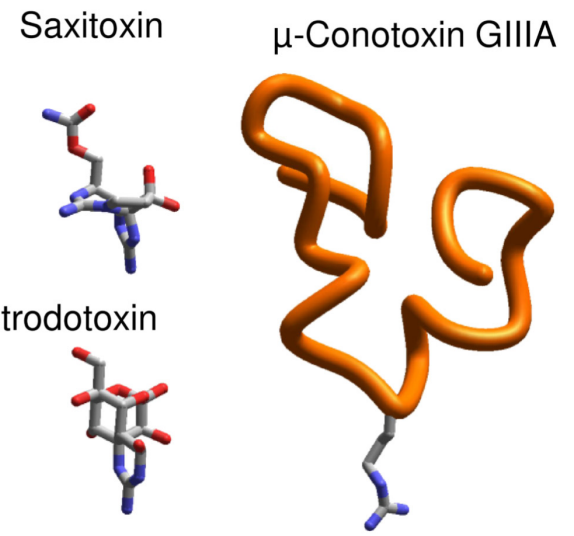

FIGURE 1 | Eukaryotic sodium channels and the outer pore blockers. (A) $\alpha$-Subunit transmembrane topology. Positions of the selectivity filter DEKA residues and the outer carboxylates, which interact with TTX, STX and $\mu$-CTX are marked by red dots. (B) General 3D model of sodium channels. Positions of toxin-interacting residues are shown by red circles. (C) $3 D$ structures of the outer pore blocking toxins. Sown is Arg13 in GIIIA, which is critical for the channel block.

Voltage-gated sodium channels play key roles in physiology of excitable cells and pathology of nerves and muscle (Hille, 2001). The sodium ions influx to the cell further depolarizes the membrane, thus generating the rising phase of the action potential. Within few milliseconds after their opening, the channels rapidly inactivate in the process known as fast inactivation. Following membrane repolarization, VGSCs recover from inactivation and the activation gate closes (Catterall, 2012). After prolonged membrane depolarization (seconds to minutes), VGSCs enter into slow-inactivated states, the process important for regulating membrane excitability, action potential patterns, and spike frequency adaptation (Vilin and Ruben, 2001; Ulbricht, 2005). Due to their widespread expression and critical functions in electrically excitable cells, VGSCs are targets for deadly toxins, which are synthesized by poisonous organisms as the attack or/and defense weapons (Catterall, 1980). Multiple diseases are associated with mutations in sodium channels (Catterall, 2014; Huang et al., 2017; Sanders et al., 2018). All these hallmarks have made VGSCs the subject of intensive studies in academia and industry. One of goals of these studies is development of various drugs including local anesthetics, antiarrhythmics, anticonvulsants, and antidepressants. The drug design and development requires high-resolution 3D structures of the channels. Thirteen years after the breakthrough publication of the KcsA potassium channel structure (Doyle et al., 1998), structure of the prokaryotic sodium channel, NavAb became available (Payandeh et al., 2011). Recently, NMR has been used to explore interactions of toxins with VSDs of the Nav1.4 channel (Mannikko et al., 2018). Cryo-EM structures of the cockroach sodium channel NavPaS (Shen et al., 2017) and electric eel Nav1.4 channel (Yan et al., 2017) provide currently the most comprehensive structures.

Despite the impressive progress in structural studies, highresolution 3D structures are still unavailable for mammalian VGSCs. Rational drug design also requires knowledge of the channel structures in different functional states (open, closed, fast-inactivated, and slow-inactivated). Furthermore, structures of the channel mutants underlying channelopathies and multiple drug-channel complexes in different channel states are unlikely to become available in the foreseeable future. This makes theoretical 
methods, which help integrate results of experimental studies and propose testable predictions, an important component of structural studies. However, computational approaches have serious limitations. Thus, docking of a ligand into a known $3 \mathrm{D}$ structure of an ion channel does not necessarily yield an unambiguous ligand-channel model. Problems that are difficult to resolve with standard computational protocols include multiplicity of possible ligand-protein complexes, unknown ionization state of residues, and missing or misinterpreted ions or water molecules. Among other factors that are difficult to take into account are membrane voltage, auxiliary subunits, and entropy component of free energy. As a result, computational studies produce sometimes controversial models of ligand-bound channels (Zimin et al., 2010; Marzian et al., 2013) despite each model is consistent with certain set of experimental data. Therefore, comparison of theoretical models with later published experimental structures is important.

Here, we review application of theoretical approaches for understanding details of the pore organization in VGSCs. We describe how integration of various experimental data, including data on toxin action allowed predicting fine details of the pore structure, including the selectivity filter geometry, which in view of the NavPaS and Nav1.4 structures proved correct.

\section{MOLECULAR PROBES OF THE SODIUM CHANNEL STRUCTURE}

Many ideas of molecular organization of VGSCs were elucidated from the analysis of the channel interactions with small molecules and peptides, whose structures are available or predictable. Even before the amino acid sequences of ion channels were published (Numa and Noda, 1986), important conclusions about the molecular organization of ion channels had been made. For example, the dimensions of the selectivity filter where proposed to match those of the largest permeant organic cations (Hille, 1971). Structure-function studies of ligands reveled the binding site organization (see Hille, 2001). Particularly, analysis of local anesthetics' action supported the conclusion on two access pathways for some drugs into the channel pore: a hydrophilic pathway from inside the cell through the open activation gate and a hydrophobic pathway from the membrane (Hille, 1977). With advent of the molecular biology era, the transmembrane topology of VGSCs was deduced from analysis of hydrophobicity (Noda and Numa, 1987).

Mutational studies helped to determine roles of individual residues in toxin binding (Terlau et al., 1991) that in turn allowed to propose a 3D scheme of toxin binding site (Lipkind and Fozzard, 1994). The era of X-ray crystallography of ion channels opened a possibility to build homology models of sodium channels and move from schematic representation to more realistic 3D homology models. The key roles in these indirect studies were played by highly specific toxins, which belong to various categories and target different sites. The channel activators (agonists) include batrachotoxin, veratridine, aconitine and grayanotoxins, which according to recent data bind inside the pore, stabilize the open state, but do not prevent the ion permeation (Tikhonov and Zhorov, 2005b; Wang et al., 2006). The mechanism of batrachotoxin action (Du et al., 2011) resembles that of a surgical stent in the blood vessel. Another group of toxins includes peptides produced by scorpions (Wang et al., 2011; Zhang J.Z. et al., 2011; Zhang J.Z. et al., 2012), spiders (Bosmans and Swartz, 2010; Minassian et al., 2014) and sea anemones (Xiao et al., 2014). These toxins bind between the pore domain and VSDs and modify the channel activation and inactivation by various toxin-specific mechanisms. Mutagenesis and modeling studies of scorpion alpha- and beta-toxins suggested that VSD-IV and VSD-II are positioned, respectively, close to the pore domain helices in repeats I and III (Leipold et al., 2006; Cohen et al., 2007; Wang et al., 2011; Chugunov et al., 2013). TTX, STX, and $\mu$-CTXs were critically important for structural studies of the outer pore (Figure 1C).

\section{TETRODOTOXIN AND SAXITOXIN}

Action of these small semi-rigid toxins on VGSCs is reviewed by many authors (e.g., Hille, 2001; Moczydlowski, 2013; Thottumkara et al., 2014). Briefly, by the early 1960s it had became clear that the "puffer fish poison" and "clam poison" contain small molecules that in nanomolar concentrations reversibly block initiation and propagation of the action potential. The blocking effects of TTX and STX on electrical excitability were systematically studied (e.g., Dettbarn et al., 1960; Narahashi et al., 1960; Evans, 1964). The chemical structures of TTX and STX were later determined (Woodward and Gougoutas, 1964; Schantz et al., 1975). TTX and STX were proposed to block the ion permeation by physically occluding the sodium channel pore (Kao and Nishiyama, 1965). Bertil Hille integrated available experimental data in a schematic model where TTX and STX blocked the sodium channel vestibule (Hille, 1975a,b). In this model, the toxin guanidinium group fit into the selectivity filter lined by six oxygen atoms that form an anionic ring of 3-5 in diameter. The model predicted at least five hydrogen bonds between the toxin and the channel. Subsequent site-directed mutagenesis of the channel protein revealed at least 10 residues in the four channel repeats whose substitutions reduce the TTX and STX affinity by more than twofold.

Intensive mutational studies revealed that TTX and STX binding sites include selectivity-filter residues in the DEKA ring and outer carboxylates three to four positions downstream from the DEKA residues (Figure 1A). In combination with structureactivity studies of toxin derivatives, the mutational analysis helped to determine pairwise contacts between the channel residues and chemical groups of TTX and STX (Figures 1A,B). In particular, the DEKA ring and outer carboxylates were found to be critical for the action of TTX and STX (Terlau et al., 1991). Later TTX and STX were shown to similarly interact with the selectivity filter residues, but differently with the outer carboxylates (Penzotti et al., 1998). Tomaselli and coauthors mapped TTX-sensing residues in P-loops by cysteine scanning (Yamagishi et al., 2001). Further studies revealed important details of TTX and STX action in different sodium channels (Choudhary et al., 2003; Bricelj et al., 2005; Jost et al., 2008; 


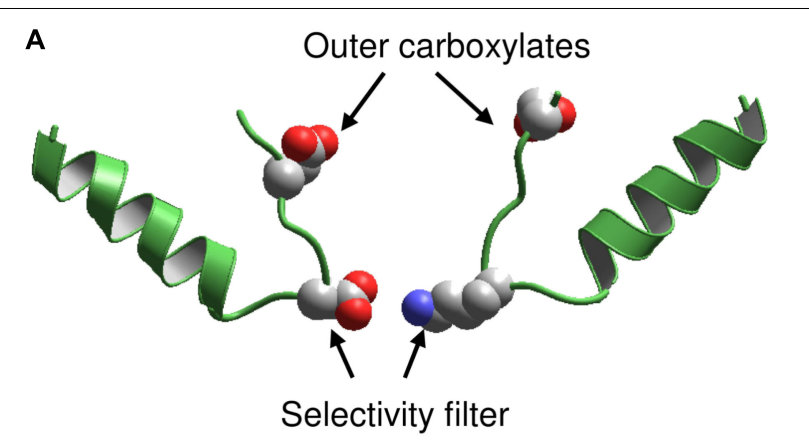

B

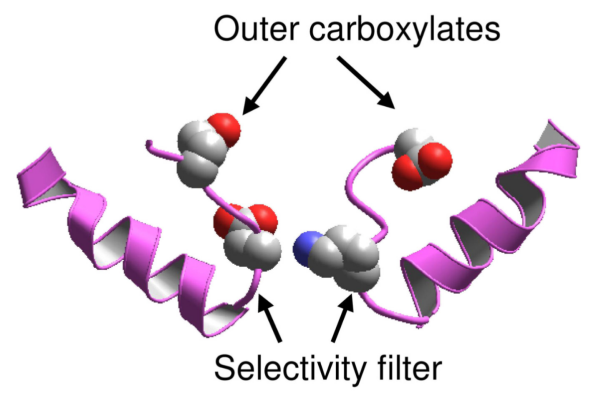

C

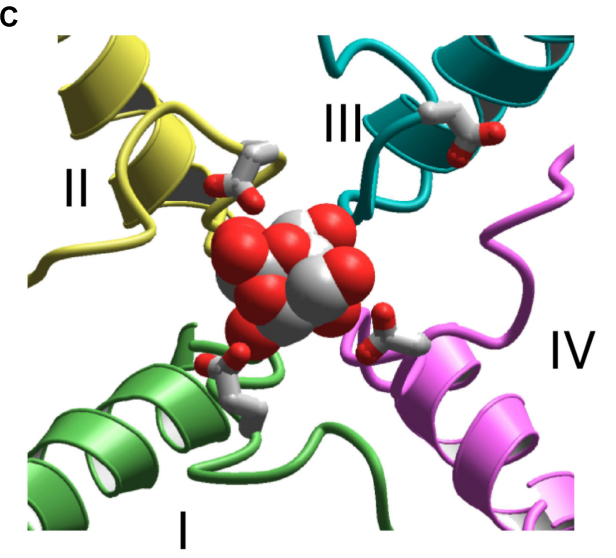

FIGURE 2 | KcsA-based models of sodium channels. (A) Model (Lipkind and Fozzard, 2000) in which the $P$ helices are shifted from the pore axis as compared with KcsA and the DEKA selectivity filter is located at the P-loop turn. (B) Model (Tikhonov and Zhorov, 2005a) in which the P-helices are disposed as in KCSA, and the DEKA ring is located in the middle of the ascending limbs. (C) Extracellular view at the TTX-bound model (Tikhonov and Zhorov, 2012). Repeats I, II, III, and IV are green, yellow, cyan, and magenta, respectively. The outer carboxylates are shown as sticks.

Conotoxins are important research tools and potential therapeutic substances. Currently, only $\sim 0.1 \%$ of conotoxins are characterized pharmacologically. Conotoxins have many different molecular targets, including various ion channels (Lewis et al., 2012). Four classes of VGSC-targeting conotoxins $(\mu, \mu \mathrm{O}$, $\delta$, and $\mathrm{L}$ ) have been isolated from cone snail venoms (Cruz et al., 1985; Fainzilber et al., 1994; McIntosh et al., 1995; Fiedler et al., 2008). These conotoxins have different mechanisms of action. Two classes ( $\mu$ and $\mu \mathrm{O}$ ) inhibit and two classes ( $\delta$ and L) activate VGSCs (Green and Olivera, 2016).

Best characterized are $\mu$-CTXs isolated from the venom of piscivorous Conus species (Green et al., 2014). The folding of $\mu$-CTXs is stabilized by three disulfide bridges arranged in a type III framework (CC-C-C-CC). The $\mu$-CTXs binding site overlaps with that of TTX and STX (Fozzard and Lipkind, 2010), but since $\mu$-CTXs are larger than TTX and STX, they exhibit greater specificity for VGSC isoforms. Most of $\mu$-CTXs are positively charged molecules, which are electrostatically attracted to the acidic residues in the VGSC outer vestibule. In several $\mu$-CTXs, a single basic residue, e.g., Arg13 in GIIIA (Figure 1C), is critical for the ion current block (Chang et al., 1998; Hui et al., 2002). Other basic residues in $\mu$-CTXs control their orientation and binding to the channel.

Mutational analysis of the channel and $\mu$-CTXs revealed their specific pairwise contacts (Chang et al., 1998; Xue et al., 2003) and the clockwise arrangement of the four repeats at the extracellular view (Dudley et al., 2000; Li et al., 2001; Choudhary et al., 2007). Furthermore, the mutant cycle determined the free energy of interactions for certain toxin-channel contacts ( $\mathrm{Li}$ et al., 2001; Choudhary et al., 2007). Binding sites of different $\mu$-CTXs essentially overlap despite the fact that patterns of residue-residue interactions are not identical. All the outer carboxylates, which interact with permeant ions (Khan et al., 2002), are key components of the binding sites for TTX, STX, and $\mu$-CTXs. Interestingly, whereas TTX and STX completely block the current, residual currents are observed in the channel complexes with some native and mutant $\mu$-CTXs (Hui et al., 2002; McArthur et al., 2011a; Wilson et al., 2011). Recently, the LRET spectroscopy was used to estimate distances between $\mu$-CTX bound in the central pore and VSDs in their resting and activates states (Kubota et al., 2017).

\section{SODIUM CHANNEL MODELS BASED ON X-RAY STRUCTURES OF POTASSIUM CHANNELS}

Walker et al., 2012). Substitutions of a tyrosine residue C-terminal to the repeat I aspartate with unnatural amino acids demonstrated a strong contribution of cation-pi interactions in TTX binding (Santarelli et al., 2007).

\section{CONOTOXINS}

More than 700 cone snail species from at least 16 genetically distinct superfamilies produce conotoxins, diverse peptides often synthesized with post-translational modifications. of the TTX and STX receptor (Lipkind and Fozzard, 1994) 4 years before the first crystal structure of an ion channel (KcsA potassium channel) was published (Doyle et al., 1998). The model employed the data that action of TTX and STX is dramatically reduced by substitutions of the selectivity-filter aspartate and glutamate, and the outer carboxylates in repeats I, II, and IV (Terlau et al., 1991). In this model, anti-parallel hairpin-like segments from the four P-loops form a funnel-like toxin binding region. The TTX guanidinium group binds to the selectivity
Lipkind and Fozzard proposed a pioneering structural model 
filter and a hydroxyl group forms an H-bond with the outer carboxylate in repeat II. TTX and STX reside in the cavity between the four hairpins and interact with other residues that, according to experimental data, are expected to contribute to the toxins binding site. At the extracellular view of this model, repeats I, II, III, and IV are arranged clockwise. This fundamental prediction has been proven with the mutant cycle analysis of the pairwise residue interactions of $\mu$-CTX GIIIA and the channel (Dudley et al., 2000).

Following publication of the KcsA X-ray structure, Lipkind and Fozzard elaborated a homology model of the Nav1.4 channel and docked TTX and STX using experimental data on toxinchannel interactions (Lipkind and Fozzard, 2000). However, the outer pore of KcsA appeared too narrow to accommodate the bulky semirigid toxins. To resolve the problem, the selectivityfilter DEKA residues were positioned at the border between the central cavity and the outer pore, at the $\mathrm{C}$-ends of P-helices (Figure 2A). The P-helices along with the outer pore-lining ascending limbs were shifted farther from the pore axis as compared to KcsA. To visualize known pairwise contacts between large $\mu$-CTXs and the channel, the KcsA-based model of Nav1.4 was further modified by shifting the P-helices even farther from the pore axis and increasing their slope relative to the pore axis (Choudhary et al., 2007).

A stronger 3D similarity between potassium and sodium channels was assumed in the alternative KcsA-based model of Nav1.4 with TTX and STX (Tikhonov and Zhorov, 2005a). In this model, the DEKA residues are placed in the middle of the ascending limbs, and the P-helices remained in positions, which are seen in KcsA (Figure 2B). Intensive Monte Carlo energy minimizations yielded the toxin-channel complexes where TTX and STX fit snugly into the outer pore (Figure 2C). Despite the different placements of the DEKA residues and different positions of P-helices, the mutual disposition of the DEKA ring and the ring of outer carboxylates are similar in both models, which were built to integrate the same set of experimental data on toxin-channel contacts.

\section{HYDROPHOBIC ACCESS PATHWAY FOR LOCAL ANESTHETICS}

Despite the fact that binding site of local anesthetics in the central cavity is far from the binding sites for TTX and $\mu$-CTXs in the outer vestibule, the toxins helped to resolve a paradox of the closed channel block by local anesthetics. That some drugs can block VGSCs by reaching their binding sites via a hydrophobic pathway was initially proposed basing on the analysis of structure-function relations of local anesthetics and related compounds (Hille, 1977). Permanently charged quaternary compounds block closed cardiac VGSCs, but not neuronal and skeletal-muscle isoforms (Alpert et al., 1989; Qu et al., 1995). Mutational data suggested critical roles of some residues in the III/IV repeat interface. Localization of this pathway between transmembrane helices IIIS6 and IVS6 and IIIP was predicted in a computational study (Tikhonov and Zhorov, 2005a). More recent computations (Boiteux et al., 2014), which

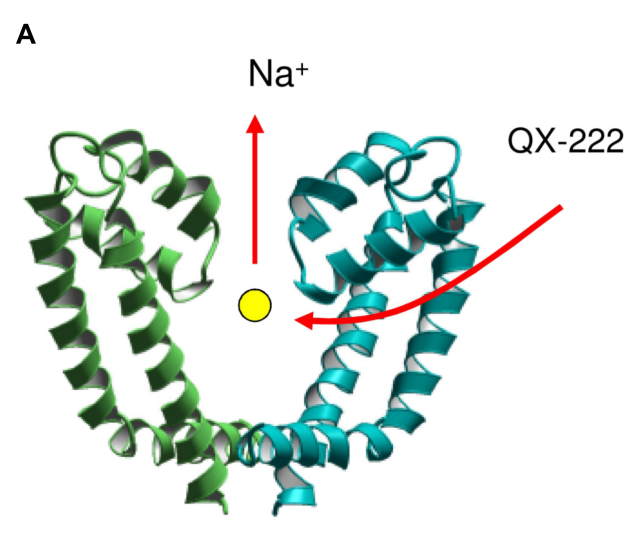

B

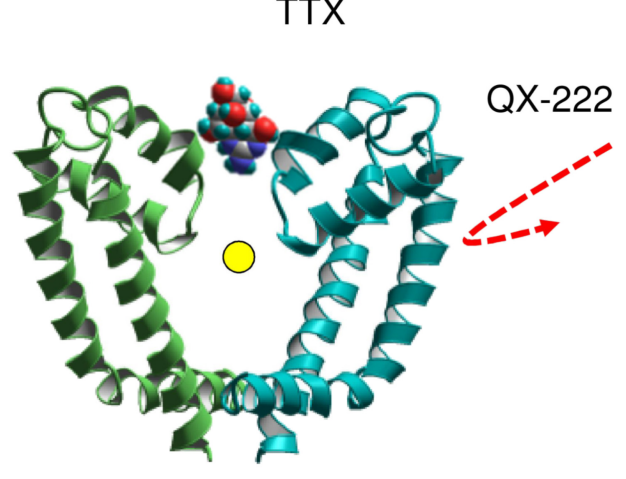

C

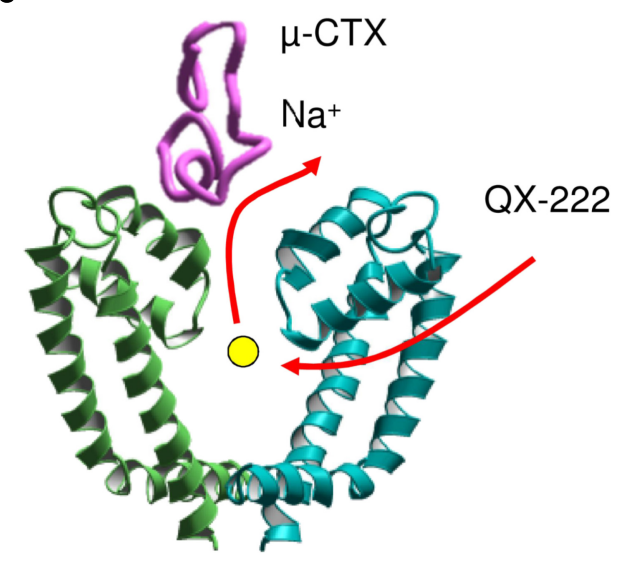

FIGURE 3 | A scheme of coupled movement of a sodium ion and ligand QX-222 in the closed cardiac sodium channel (Bruhova et al., 2008). (A) The permanently charged QX-222 reaches the inner pore via III/IV repeat interface and displaces the resident ion that leaves the central cavity through the selectivity filter. (B) When the outer pore is blocked by TXX, the sodium ion is trapped in the central cavity and prevents binding of QX-222. (C) A $\mu$-CTX mutant does not completely block the sodium current. The sodium ion can escape through the incompletely sealed outer pore and vacate the cation-attractive central cavity for binding of QX-222.

used the X-ray structure of bacterial sodium channel NavAb (Payandeh et al., 2011) agree with this prediction. 
However, it was unclear why TTX (Qu et al., 1995), but not a GIIIA mutant (Sunami et al., 2001) prevents the closed channel block by a permanently charged local anesthetic-like ligand, QX-314. At first sight, this observation suggests that the access pathway to the closed channel involves the outer pore. A solution of this paradox was proposed in a model (Figure 3) that combines two features: (i) the access pathway for local anesthetics into the closed channel through the membraneexposed III//IV interface and (ii) a sodium ion residing in the central cavity of the TTX-bound channel (Bruhova et al., 2008). The electronegative focus of $\mathrm{P} 1$ helices would be attractive for cationic local anesthetics unless it is occupied by another cation. Thus, the permanently charged drug targeting the cavity should displace from it the resident sodium ion (Figure 3A). In the TTXbound closed channel, the sodium ion residing in the central cavity lacks any way to leave it: the activation gate is closed, the hydrophobic pathway is prohibitive for the hydrated ion, and the outer-pore route is blocked by TTX. Therefore, quaternary compounds cannot reach the binding site due to repulsion from the ion (Figure 3B). When the GIIIA mutant R13N binds to the channel, some residual current is observed (Sunami et al., 2001). Therefore, when the local anesthetic displaces the sodium ion, it can escape the central cavity to the extracellular space by moving between polar residues of the channel and the GIIIA mutant (Figure 3C).

\section{PROGRESS INSPIRED BY X-RAY STRUCTURES OF PROKARYOTIC SODIUM CHANNELS}

In the X-ray structures of bacterial sodium channels (Payandeh et al., 2011; Zhang X. et al., 2012) the selectivity filter glutamates are in the middle of the ascending limbs and positions of P1-helices are close to those in potassium channels. These experimental structures supported the assumption on close $3 \mathrm{D}$ similarity of membrane-descending P-helices in sodium and potassium channels (Tikhonov and Zhorov, 2005a). Furthermore, the X-ray structures demonstrated wide fenestrations between subunits that could provide a spacious access pathway for some ligands from the membrane into the inner pore. Thus, the predicted localization of the hydrophobic access pathway for some drugs (Tikhonov and Zhorov, 2005a; Bruhova et al., 2008) has been also confirmed.

An important model-based prediction was presence of a sodium ion at the cation-attractive focus of P1 helices (Tikhonov et al., 2006). In proposed models this ion lacks direct contacts with the channel residues, and binds to various ligands including batrachotoxin (Tikhonov and Zhorov, 2005b; Du et al., 2011), local anesthetics and anticonvulsants (Tikhonov et al., 2006; Bruhova et al., 2008; Tikhonov and Zhorov, 2017b; Buyan et al., 2018). Now a completely hydrated sodium ion $\mathrm{Na}_{\text {III }}$ is seen in the focus of four backbone carbonyls at the border of the outer pore and the inner pore, close to the focus of P1 helices (Naylor et al., 2016). Such location makes the ion available for direct contacts with ligands targeting the central cavity.

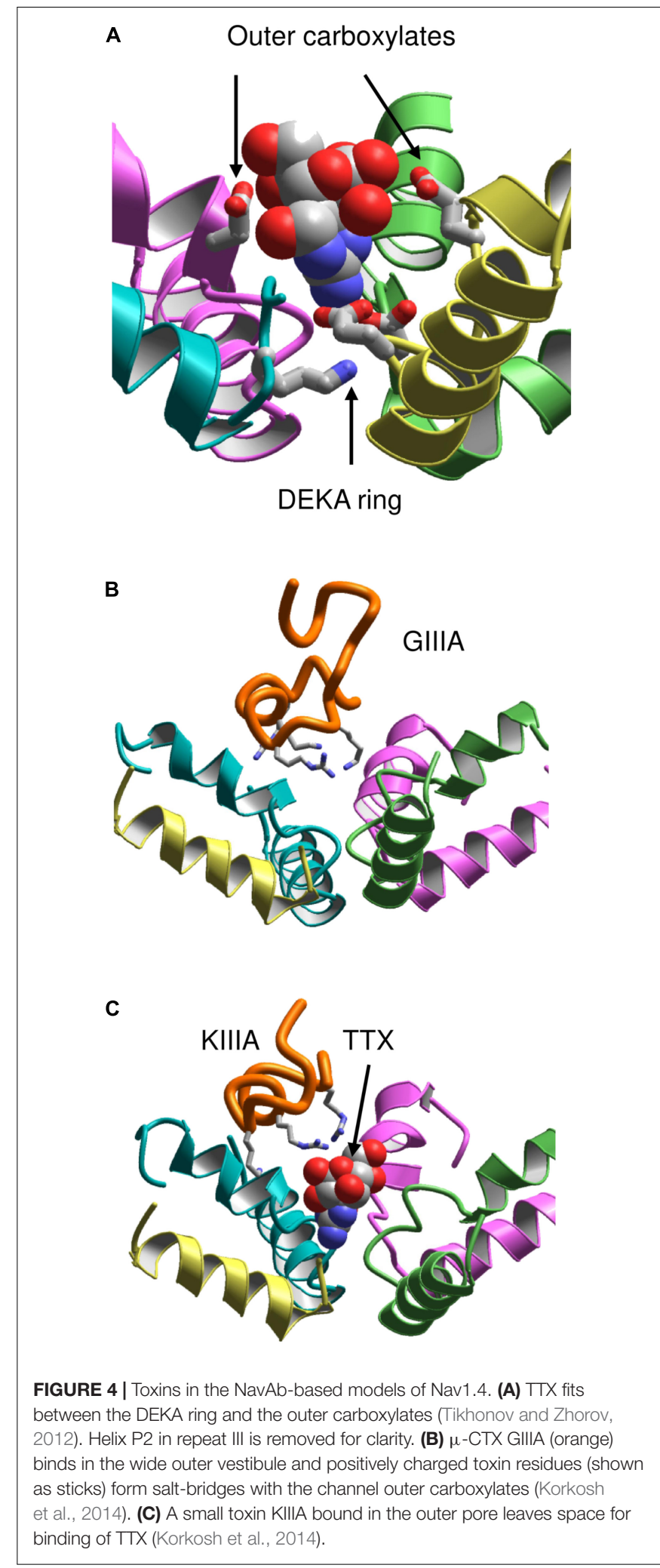

It should be noted that models based on the X-ray structures of potassium channels failed to predict that the C-terminal halves of P-loops contain membrane-ascending P2 helices, which make the 


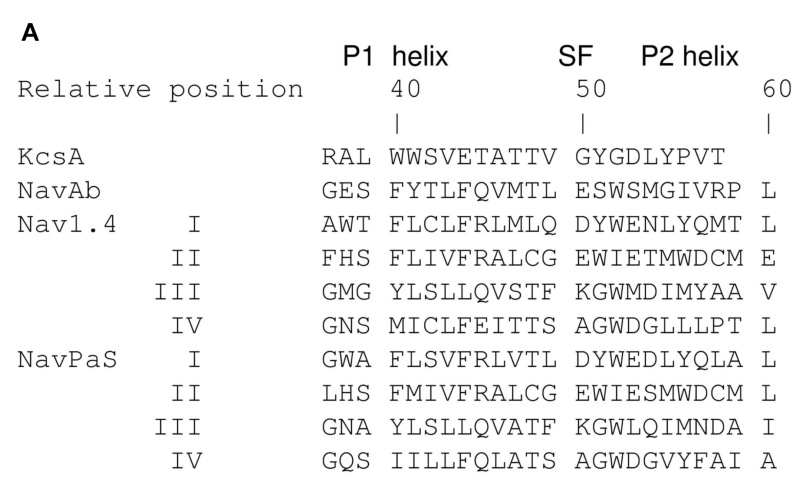

B

\section{NavPaS vs. Nav1.4}
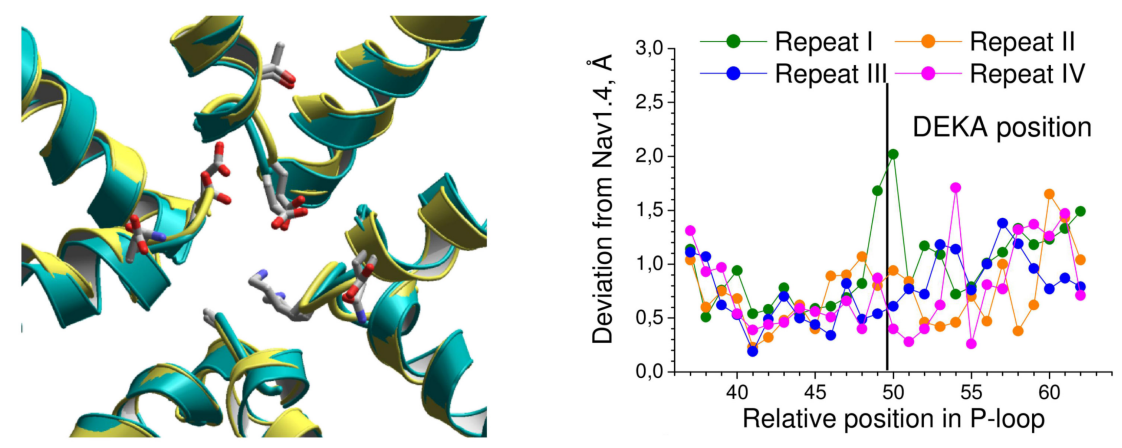

C
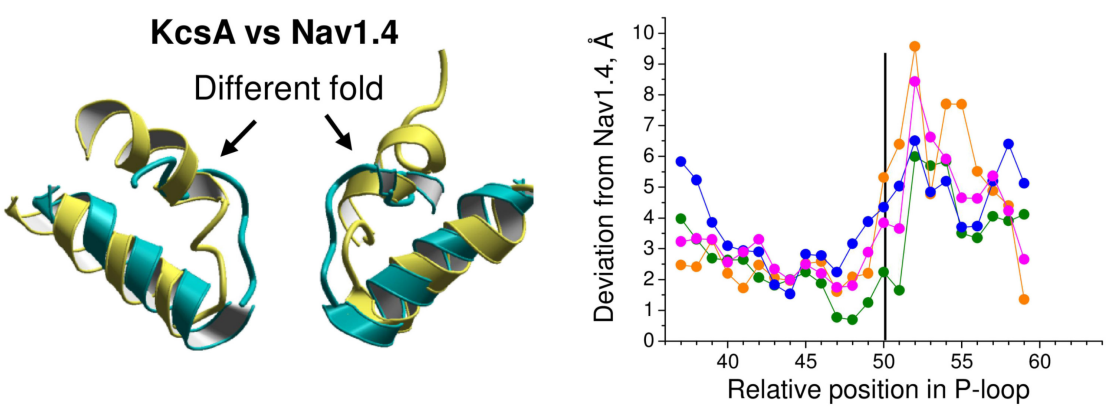

D

NavAb vs Nav1.4
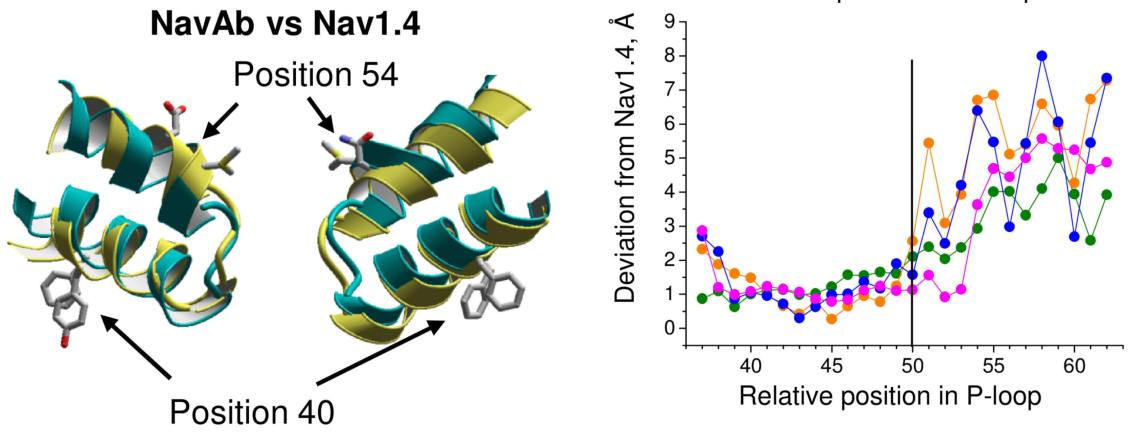

FIGURE 5 | Comparison of P-loops in Nav1.4, NavPaS, KcsA, NavAb. (A) Straightforward sequence alignment of P-loops. In the NavPaS and Nav1.4 sequences the repeat numbers are indicated by roman numerals. The top row shows relative positions of residues in the aligned sequences; position 50 is assigned to the selectivity-filter DEKA residues. Comparison of NavPaS (B), KcsA (C), and NavAb (D) structures with Nav1.4. The structures are 3D aligned by minimizing RMS deviations of alpha carbons in the P1 helices from matching atoms in Nav1.4 according to the sequence alignment shown in (A). Left panels show 3D aligned structures with Nav1.4 in yellow. Right panels are plots of deviations of alpha carbons from matching atoms in Nav1.4. (B) NavPaS is similar to Nav1.4 and most deviations are less than $1.5 \AA$. The selectivity filter residues and the outer carboxylates are shown as sticks in the superimposed structures. (C) KcsA significantly deviates from Nav1.4 in the outer pore region where sodium and potassium channels have different folding. (D) NavAb and Nav1.4 have similar P1-helices, but large deviations are seen in the P2 helices where amino acids in matching positions (according to the straightforward sequence alignment) have different orientations in the 3D-aligned structures. 
A

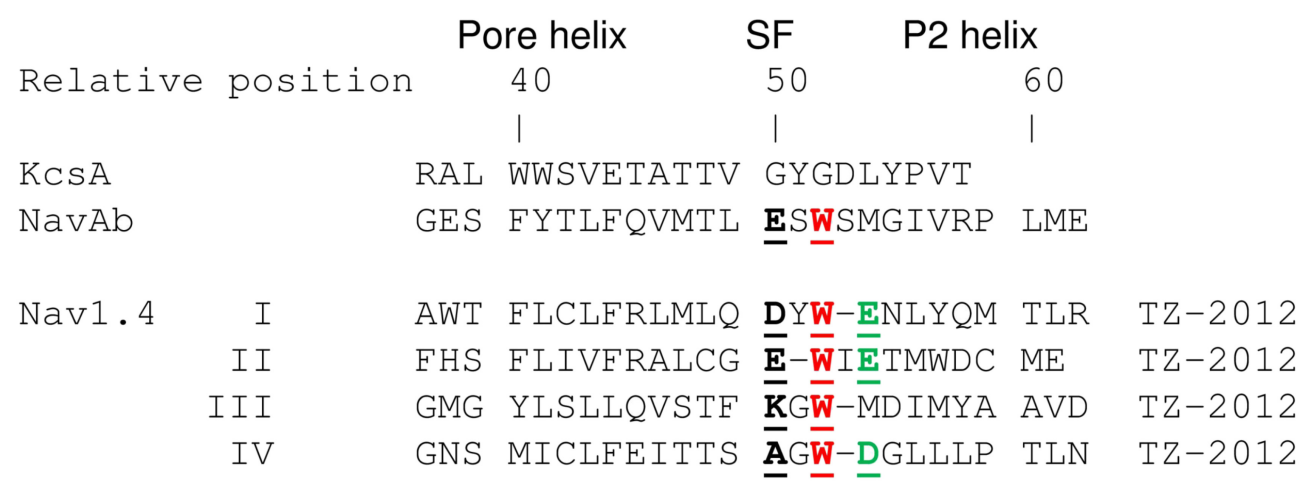

Nav1.4 II FHS FLIVFRALCG $\underline{\mathbf{E}-W}$ IE-TMWD CME Current work

B
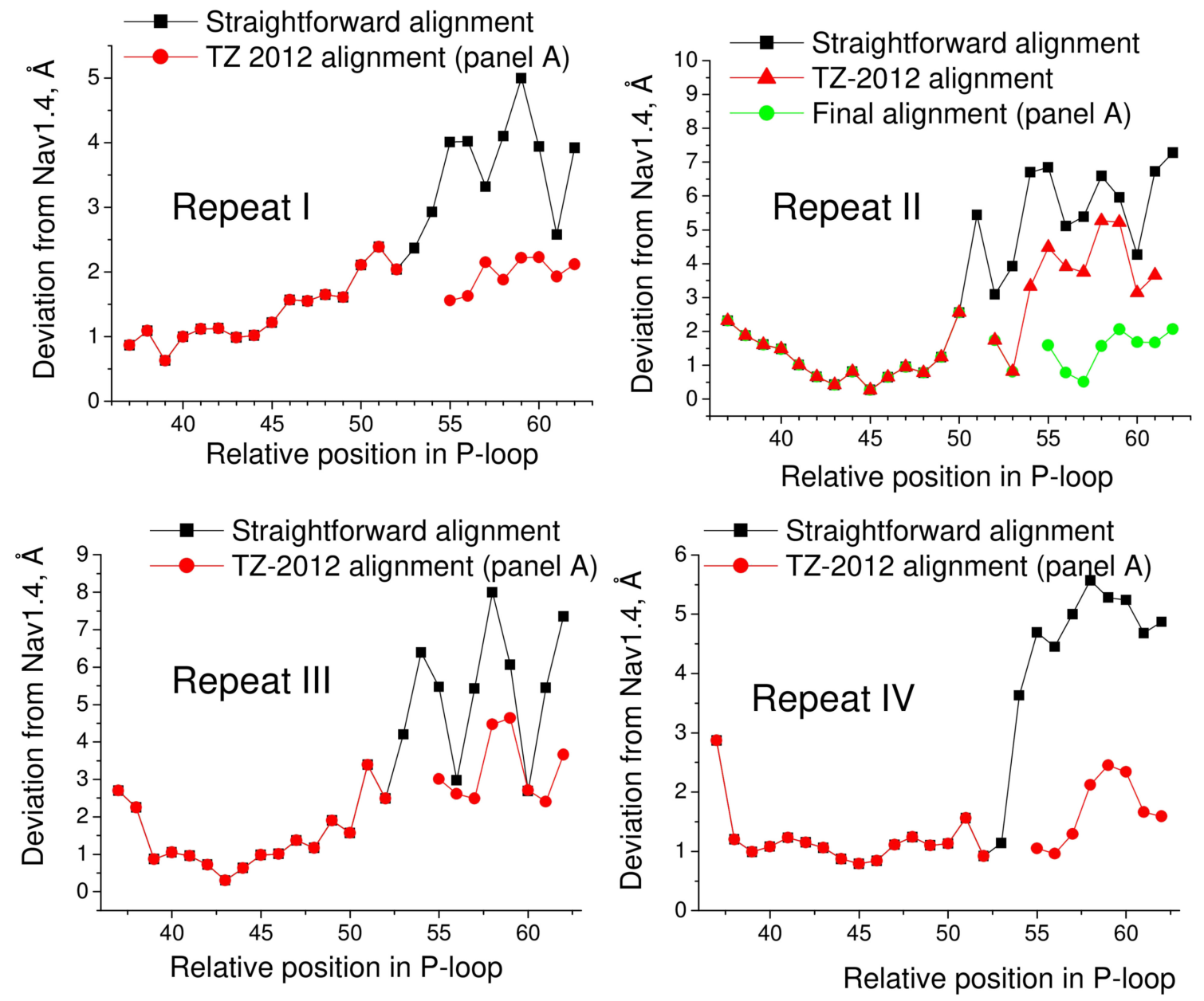

FIGURE 6 | Adjusted sequence alignment decreases deviations of matching atoms in 3D aligned structure of NavAb. (A) Adjusted sequence alignment (TZ-2012) of P-loops in KcsA, NavAb, and Nav1.4 (Tikhonov and Zhorov, 2012). Highlighted are residues in the DEKA locus, conserved tryptophans that stabilize folding of the P-loops, and the outer carboxylates. In the adjusted, but not the straightforward (Figure 5A) sequence alignment the conserved tryptophans are in the matching positions. The second deletion in repeat II of Nav1.4 is introduced in the current work to minimize deviations between matching alpha carbons in the P2 helices of NavAb and Nav1.4. (B) Deviation of alpha carbons in repeats I-IV of Nav1.4 from the NavAb atoms, which are considered as matching according different sequence alignments. Introducing deletions decreases deviations and smoothes the deviation curves. 

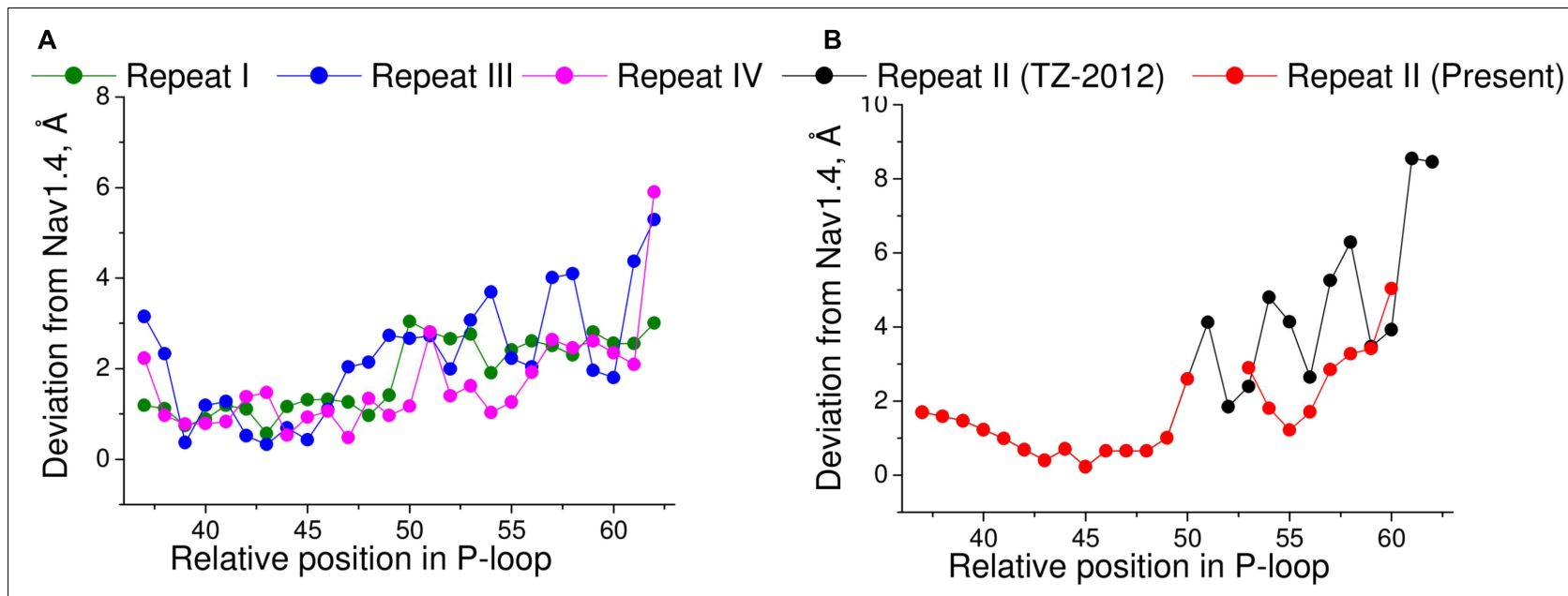

C

D
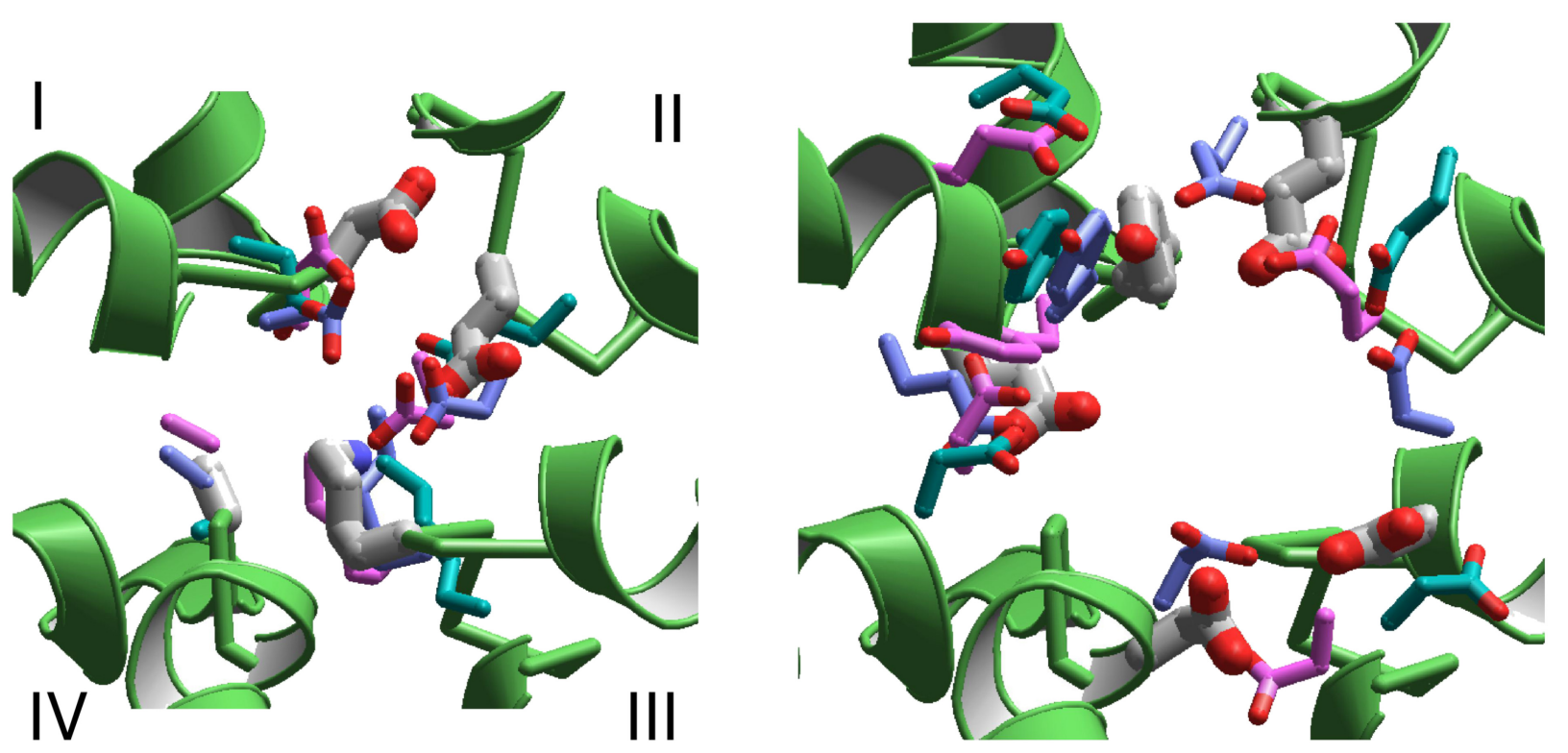

FIGURE 7 | Comparison of the Nav1.4 cryo-EM structure with homology models. (A) P-loop alpha carbons in repeats I, III, and IV of the NavAb-based model (Tikhonov and Zhorov, 2012) are close to the matching atoms in the Nav1.4 structure. (B) In repeat II, big irregular deviations are seen because the Nav1.4 sequence aligned with NavAb lacks deletion at position 54 (Figure 6A). Introducing this deletion reduced deviations and the curve becomes similar to those in other repeats. (C) Side chains of the DEKA ring in 3D aligned structures. Only the Nav1.4 backbone is shown. The Nav1.4 residues are shown as thick sticks. Residues in the KcsA-based models (Lipkind and Fozzard, 2000; Tikhonov and Zhorov, 2005a) and NavAb-based model (Tikhonov and Zhorov, 2012) are shown, respectively, with cyan, magenta, and violet thin sticks. (D) Side chains of the outer carboxylates and a TTX-interacting tyrosine in position 51 of repeat I. Residues are rendered as in (C).

outer pore wide enough to accommodate large toxins. Therefore, the NavAb structure motivated new theoretical studies aimed to rationalize binding of toxins to the outer pore. However, a NavAb-based model of Nav1.4 built using the straightforward sequence alignment of P-loops (Tikhonov and Zhorov, 2012) failed to explain mutational data on TTX interactions with the outer carboxylates, which faced away the pore axis and did not interact with the toxin. To resolve the problem, deletions near the DEKA residues were introduced in the Nav1.4 sequence aligned with NavAb (Tikhonov and Zhorov, 2012).
The model built with this alignment re-oriented the outer carboxylates so that their contacts with TTX (Figure 4A) are consistent with experimental data summarized by Lipkind and Fozzard.

This Nav1.4 model was further employed to dock $\mu$ CTXs GIIIA, PIIIA, and KIIIA (Korkosh et al., 2014). The distance constraints between GIIIA and the channel residues, which represent pairwise interactions revealed by the mutant cycle analysis (Choudhary et al., 2007), facilitated the toxin docking. These constraints have been satisfied without deforming the toxin 
and the channel backbones (Figure 4B). Correlation between the computed and experimental energies of specific interactions (Choudhary et al., 2007) further validated the model. The voltagedependence of action of PIIIA and its mutants helped determine the depths of individual residues in the membrane electric field (McArthur et al., 2011b). The model of PIIIA-Nav1.4 complex (Korkosh et al., 2014) is in a good agreement with these data.

Some native and mutant conotoxins incompletely block the current (Hui et al., 2002; McArthur et al., 2011a; Wilson et al., 2011). For the first time the model (Korkosh et al., 2014) proposed the following rationale for this interesting phenomenon: TTX fits the narrow part of the outer pore and completely plugs it, whereas large $\mu$ CTXs bind above the outer pore and cover, but do not plug it. The ion permeation is blocked by the $\mu$ CTX charged residues that form salt bridges with the outer carboxylates. If the channel-bound toxin lacks some of the basic residues, at least one carboxylate would not form a salt bridge with the toxin and provide a transient binding site for permeating ions. The latter would pass through the outer pore of toxin-bound channel, although the ion current would be smaller than that in toxinfree channels. Intriguingly, TTX and KIIIA can simultaneously bind to the sodium channel (Zhang et al., 2009; Van Der Haegen et al., 2011; Wilson et al., 2011; Khoo et al., 2012; Stevens et al., 2012). In agreement with these data, the model simultaneously accommodates both TTX and KIIIA (Korkosh et al., 2014) (Figure 4C).

\section{HOMOLOGY MODELS vS. CRYO-EM STRUCTURES OF EUKARYOTIC SODIUM CHANNELS}

The above homology models have been built using certain assumptions and experimental data whose interpretations are ambiguous. Furthermore, precision of computational models of complex transmembrane proteins is limited. Given these limitations, correctness of the homology models was questionable. Now the cryo-EM structures of VGSCs allow judging the accuracy of the modeling predictions. To obtain the accuracy criteria, we first compared 3D aligned structures of Nav1.4 with NavPaS, KcsA, and NavAb. The 3D alignment was obtained by minimizing RMS deviations of alpha carbon atoms in residues, which according to the straightforward sequence alignment are in matching positions of the P1 helices (Figure 5A). The P1 helices are chosen for the $3 \mathrm{D}$ alignment because they are the most structurally conserved segments of P-loop channels (Tikhonov and Zhorov, 2017a).

The 3D aligned NavPaS and Nav1.4 are very similar and most deviations between matching atoms do not exceed $1.5 \AA$ (Figure 5B). The largest deviations correspond to the selectivity filter aspartate in repeat I and the outer carboxylate in repeat IV. The deviations generally increase at the N-end of P1 and C-end of P2 where the helices are connected to flexible loops. Functional groups at the ends of long flexible sidechains are expected to deviate more than alpha carbons. Indeed, distances between terminal groups of the residues, which contribute to the TTX binding site, vary from 0.7 to $2.7 \AA$ (Table 1). We further compared Nav1.4 with KcsA and NavAb whose X-ray structures were used as templates to model eukaryotic sodium channels. The P1 helices in potassium and sodium channels have different slope relative to the pore axis and the deviation distances between alpha carbons of matching residues vary from 1 to $3 \AA$. The distances sharply increase to 5-9 A at the outer-pore region, which folds differently in sodium and potassium channels (Figure 5C).

The P1 helices in the 3D aligned NavAb and Nav1.4 are similar and most deviations are less than $2 \AA$. Importantly, the four deviation curves in this region are smooth (Figure 5D). However, downstream the selectivity filter (position 50) the deviations sharply increase, resembling those between the $3 \mathrm{D}$ aligned Nav1.4 and KcsA. In this region, the deviation curves become highly irregular. Similar irregular curves are seen in the KcsA/Nav1.4 deviation plot at positions C-terminal to the selectivity filter where P-loops of these channels have different folding (Figure 5C). However, the irregular curves in the $\mathrm{NavAb} / \mathrm{Nav1.4}$ deviation plot are surprising because the folding of P1 and P2 helices in these channels is very similar. The cause of the irregular deviation curves is the straightforward sequence alignment downstream the selectivity filter. Figure 5D shows that residues in the matching position of $\mathrm{P} 1$ helices have similar $3 \mathrm{D}$ orientations, whereas orientations of residues in the P2 helices

TABLE 1 | Deviations (Å) of central atoms of TTX-binding functional groups in homology models and NavPaS from matching atoms in the Nav1.4 cryo-EM structure.

\begin{tabular}{|c|c|c|c|c|}
\hline Residue & \multicolumn{4}{|c|}{ Model/structure } \\
\hline$Y^{1051}$ & 3.7 & 6.1 & 2.2 & 0.5 \\
\hline$E^{1 p 53}$ & 10.3 & 8.1 & 2.4 & 0.7 \\
\hline$E^{2 p 53}$ & 5.4 & 4.6 & 3.9 & 2.7 \\
\hline$D^{3 p 54}$ & 4.6 & 6.6 & 6.1 & 2.4 \\
\hline$E^{2 p 50}$ & 2.9 & 2.7 & 1.9 & 0.8 \\
\hline $\mathrm{K}^{3 \mathrm{p} 50}$ & 1.1 & 1.6 & 1.5 & 2.0 \\
\hline$A^{4 p 50}$ & 1.7 & 2.1 & 1.4 & 0.4 \\
\hline
\end{tabular}


are dramatically different. To reconcile the sequence and $3 \mathrm{D}$ alignments, we introduced in the Nav1.4 sequence deletions (Figure 6A), which were proposed to build our model of TTXbound Nav1.4 (Tikhonov and Zhorov, 2012). These adjustments removed the curve irregularity and sharply decreased deviations in repeats I, III, and IV (Figure 6B). However, in repeat II significant irregularity remained after position 54, indicating another problem in the alignment. Introducing the second deletion in repeat II of Nav1.4 solved the problem (green curve in Figure 6B).

Next, we compared the Nav1.4 structure with three homology models, which have been built to rationalize action of TTX and STX. Deviations from Nav1.4 are large (4-5 $)$ in the model, which was built using the KcsA template modified to shift apart the P1 helices (Lipkind and Fozzard, 2000). Deviations of another KcsA-based model (Tikhonov and Zhorov, 2005a) from Nav1.4 are smaller, but they are still rather large $(2-3 \AA)$. The NavAb-based model (Tikhonov and Zhorov, 2012) is significantly more precise (Figures 7A,B). The largest deviations are seen C-terminal to position 54 of repeat II (Figure 7B), where necessity of the second deletion (Figure 6) was not recognized in lack of experimental data on TTX interactions with respective residues.

The above deviations of alpha carbons in the models from Nav1.4 obviously depend on the templates used for modeling. We further compared coordinates of the functional groups that interact with TTX, which served as a molecular probe to build the models. The general disposition of the TTXinteracting functional groups in all the models is similar to that in the experimental structure of Nav1.4 (Figures 7C,D). The outer carboxylates in the KcsA-based models (Lipkind and Fozzard, 2000; Tikhonov and Zhorov, 2005a) have rather large deviations from Nav1.4, whereas the NavAbbased model (Tikhonov and Zhorov, 2012) is much more precise (Table 1). The main outlier is the outer carboxylate in repeat III, which does not interact with TTX (Terlau et al., 1991) and therefore the models had been built without specific constraints between TTX and this carboxylate. Deviations of the functional groups in the NavAb-based model (Tikhonov and Zhorov, 2005a) are usually larger than respective deviations between NavPaS and Nav1.4. Of primary importance, however, is the fact that the deviations between two experimental structures of closely related eukaryotic sodium channels are in the same range as deviations of

\section{REFERENCES}

Alpert, L. A., Fozzard, H. A., Hanck, D. A., and Makielski, J. C. (1989). Is there a second external lidocaine binding site on mammalian cardiac cells? Am. J. Physiol. 257, H79-H84. doi: 10.1152/ajpheart.1989.257.1.H79

Boiteux, C., Vorobyov, I., French, R. J., French, C., Yarov-Yarovoy, V., and Allen, T. W. (2014). Local anesthetic and antiepileptic drug access and binding to a bacterial voltage-gated sodium channel. Proc. Natl. Acad. Sci. U.S.A. 111, 13057-13062. doi: 10.1073/pnas.1408710111

Bosmans, F., and Swartz, K. J. (2010). Targeting voltage sensors in sodium channels with spider toxins. Trends Pharmacol. Sci. 31, 175-182. doi: 10.1016/j.tips.2009. 12.007 the NavAb-based model from the experimental structure (Table 1).

\section{CONCLUSION}

Here we compared homology models Nav1.4, which are built using TTX, STX and $\mu$-CTXs as molecular probes, with cryo-EM structures of eukaryotic sodium channels NavPaS and Nav1.4. Due to above-mentioned limitations of indirect approaches, including homology modeling, the exact predictions are hardly possible in lack of appropriate template structures. Nevertheless, several important structural features of sodium channel pore are predicted correctly. First, the predicted clockwise arrangement of the channel repeats at the extracellular view (Dudley et al., 2000) is now seen in NavPaS and Nav1.4. Second, the general configuration of the outer pore and mutual disposition of the selectivity filter residues and outer carboxylates in the KcsA-based models (Lipkind and Fozzard, 2000; Tikhonov and Zhorov, 2012) is correctly predicted despite the outer pore structure and the selectivity filter details in KcsA are dramatically different from those in Nav1.4. Third, docking of TTX into the NavAb-based model of the Nav1.4 channel allowed improving the sequence alignment between the prokaryotic and eukaryotic sodium channels. Fourth, experimental data on action of toxins helped to predict important features of sodium channels such as localization of the hydrophobic access pathway and presence of a hydrated sodium ion in the central cavity. Thus, experimental confirmation of predictions from structural studies of ion channels, in which toxins and other small molecules were used as molecular probes, justifies employment of this approach for future research.

\section{AUTHOR CONTRIBUTIONS}

All authors listed have made a substantial, direct and intellectual contribution to the work, and approved it for publication.

\section{FUNDING}

This study was supported by Federal Agency of Scientific Organizations for IEPhB RAS.

Bricelj, V. M., Connell, L., Konoki, K., Macquarrie, S. P., Scheuer, T., Catterall, W. A., et al. (2005). Sodium channel mutation leading to saxitoxin resistance in clams increases risk of PSP. Nature 434, 763-767. doi: 10.1038/nature03415

Bruhova, I., Tikhonov, D. B., and Zhorov, B. S. (2008). Access and binding of local anesthetics in the closed sodium channel. Mol. Pharmacol. 74, 1033-1045. doi: $10.1124 / \mathrm{mol} .108 .049759$

Buyan, A., Sun, D., and Corry, B. (2018). Protonation state of inhibitors determines interaction sites within voltage-gated sodium channels. Proc. Natl. Acad. Sci. U.S.A. 115, E3135-E3144. doi: 10.1073/pnas.1714131115

Catterall, W. A. (1980). Neurotoxins that act on voltage-sensitive sodium channels in excitable membranes. Annu. Rev. Pharmacol. Toxicol. 20, 15-43. doi: 10. 1146/annurev.pa.20.040180.000311 
Catterall, W. A. (2012). Voltage-gated sodium channels at 60: structure, function and pathophysiology. J. Physiol. 590, 2577-2589. doi: 10.1113/jphysiol.2011. 224204

Catterall, W. A. (2014). Sodium channels, inherited epilepsy, and antiepileptic drugs. Annu. Rev. Pharmacol. Toxicol. 54, 317-338. doi: 10.1146/annurevpharmtox-011112-140232

Chang, N. S., French, R. J., Lipkind, G. M., Fozzard, H. A., and Dudley, S. Jr. (1998). Predominant interactions between mu-conotoxin Arg-13 and the skeletal muscle $\mathrm{Na}+$ channel localized by mutant cycle analysis. Biochemistry 37, 4407-4419. doi: 10.1021/bi9724927

Choudhary, G., Aliste, M. P., Tieleman, D. P., French, R. J., and Dudley, S. C. Jr. (2007). Docking of mu-conotoxin GIIIA in the sodium channel outer vestibule. Channels 1, 344-352. doi: 10.4161/chan.5112

Choudhary, G., Yotsu-Yamashita, M., Shang, L., Yasumoto, T., and Dudley, S. C. Jr. (2003). Interactions of the C-11 hydroxyl of tetrodotoxin with the sodium channel outer vestibule. Biophys. J. 84, 287-294. doi: 10.1016/S0006-3495(03) 74849-8

Chugunov, A. O., Koromyslova, A. D., Berkut, A. A., Peigneur, S., Tytgat, J., Polyansky, A. A., et al. (2013). Modular organization of alpha-toxins from scorpion venom mirrors domain structure of their targets, sodium channels. J. Biol. Chem. 288, 19014-19027. doi: 10.1074/jbc.M112. 431650

Cohen, L., Ilan, N., Gur, M., Stuhmer, W., Gordon, D., and Gurevitz, M. (2007). Design of a specific activator for skeletal muscle sodium channels uncovers channel architecture. J. Biol. Chem. 282, 29424-29430. doi: 10.1074/ jbc.M704651200

Cruz, L. J., Gray, W. R., Olivera, B. M., Zeikus, R. D., Kerr, L., Yoshikami, D., et al. (1985). Conus geographus toxins that discriminate between neuronal and muscle sodium channels. J. Biol. Chem. 260, 9280-9288.

Dettbarn, W. D., Higman, H., Rosenberg, P., and Nachmansohn, D. (1960). Rapid and reversible block of electrical activity by powerful marine biotoxins. Science 132, 300-301. doi: 10.1126/science.132.3422.300-a

Doyle, D. A., Morais Cabral, J., Pfuetzner, R. A., Kuo, A., Gulbis, J. M., Cohen, S. L., et al. (1998). The structure of the potassium channel: molecular basis of K+ conduction and selectivity. Science 280, 69-77. doi: 10.1126/science.280. 5360.69

Du, Y., Garden, D. P., Wang, L., Zhorov, B. S., and Dong, K. (2011). Identification of new batrachotoxin-sensing residues in segment IIIS6 of the sodium channel. J. Biol. Chem. 286, 13151-13160. doi: 10.1074/jbc.M110.208496

Dudley, S. C. Jr., Chang, N., Hall, J., Lipkind, G., Fozzard, H. A., and French, R. J. (2000). mu-conotoxin GIIIA interactions with the voltage-gated $\mathrm{Na}(+)$ channel predict a clockwise arrangement of the domains. J. Gen. Physiol. 116, 679-690. doi: 10.1085 /jgp.116.5.679

Evans, M. H. (1964). Paralytic effects of "paralytic shellfish poison" on frog nerve and muscle. Br. J. Pharmacol. Chemother. 22, 478-485. doi: 10.1111/j.14765381.1964.tb01702.x

Fainzilber, M., Kofman, O., Zlotkin, E., and Gordon, D. (1994). A new neurotoxin receptor site on sodium channels is identified by a conotoxin that affects sodium channel inactivation in molluscs and acts as an antagonist in rat brain. J. Biol. Chem. 269, 2574-2580.

Fiedler, B., Zhang, M. M., Buczek, O., Azam, L., Bulaj, G., Norton, R. S., et al. (2008). Specificity, affinity and efficacy of iota-conotoxin RXIA, an agonist of voltage-gated sodium channels $\mathrm{Na}(\mathrm{V}) 1.2,1.6$ and 1.7. Biochem. Pharmacol. 75, 2334-2344. doi: 10.1016/j.bcp.2008.03.019

Fozzard, H. A., and Lipkind, G. M. (2010). The tetrodotoxin binding site is within the outer vestibule of the sodium channel. Mar. Drugs 8, 219-234. doi: 10.3390/ md8020219

Green, B. R., Bulaj, G., and Norton, R. S. (2014). Structure and function of mu-conotoxins, peptide-based sodium channel blockers with analgesic activity. Future Med. Chem. 6, 1677-1698. doi: 10.4155/fmc. 14.107

Green, B. R., and Olivera, B. M. (2016). Venom peptides from cone snails: pharmacological probes for voltage-gated sodium channels. Curr. Top. Membr. 78, 65-86. doi: 10.1016/bs.ctm.2016.07.001

Hille, B. (1971). The permeability of the sodium channel to organic cations in myelinated nerve. J. Gen. Physiol. 58, 599-619. doi: 10.1085/jgp.58. 6.599
Hille, B. (1975a). An essential ionized acid group in sodium channels. Fed Proc. 34, 1318-1321. doi: 10.1007/978-1-4684-2637-3_2

Hille, B. (1975b). The receptor for tetrodotoxin and saxitoxin. Struct. Hypothes. Biophys. J. 15, 615-619.

Hille, B. (1977). Local anesthetics: hydrophilic and hydrophobic pathways for the drug-receptor reaction. J. Gen. Physiol. 69, 497-515. doi: 10.1085/jgp.69.4.497

Hille, B. 2001. Ion Channels of Excitable Membranes. 3rdEdn, Sunderland, MA: Sinauer Associates, 814.

Huang, W., Liu, M., Yan, S. F., and Yan, N. (2017). Structure-based assessment of disease-related mutations in human voltage-gated sodium channels. Protein Cell 8, 401-438. doi: 10.1007/s13238-017-0372-Z

Hui, K., Lipkind, G., Fozzard, H. A., and French, R. J. (2002). Electrostatic and steric contributions to block of the skeletal muscle sodium channel by mu-conotoxin. J. Gen. Physiol. 119, 45-54. doi: 10.1085/jgp.119.1.45

Jost, M. C., Hillis, D. M., Lu, Y., Kyle, J. W., Fozzard, H. A., and Zakon, H. H. (2008). Toxin-resistant sodium channels: parallel adaptive evolution across a complete gene family. Mol. Biol. Evol. 25, 1016-1024. doi: 10.1093/molbev/ msn025

Kao, C. Y., and Nishiyama, A. (1965). Actions of saxitoxin on peripheral neuromuscular systems. J. Physiol. 180, 50-66.

Khan, A., Romantseva, L., Lam, A., Lipkind, G., and Fozzard, H. A. (2002). Role of outer ring carboxylates of the rat skeletal muscle sodium channel pore in proton block. J. Physiol. 543, 71-84. doi: 10.1113/jphysiol.2002.021014

Khoo, K. K., Gupta, K., Green, B. R., Zhang, M. M., Watkins, M., Olivera, B. M., et al. (2012). Distinct disulfide isomers of mu-conotoxins KIIIA and KIIIB block voltage-gated sodium channels. Biochemistry 51, 9826-9835. doi: 10.1021/ bi301256s

Korkosh, V. S., Zhorov, B. S., and Tikhonov, D. B. (2014). Folding similarity of the outer pore region in prokaryotic and eukaryotic sodium channels revealed by docking of conotoxins GIIIA, PIIIA, and KIIIA in a NavAb-based model of Nav1.4. J. Gen. Physiol. 144, 231-244. doi: 10.1085/jgp.201411226

Kubota, T., Durek, T., Dang, B., Finol-Urdaneta, R. K., Craik, D. J., Kent, S. B., et al. (2017). Mapping of voltage sensor positions in resting and inactivated mammalian sodium channels by LRET. Proc. Natl. Acad. Sci. U.S.A. 114, E1857-E1865. doi: 10.1073/pnas.1700453114

Leipold, E., Hansel, A., Borges, A., and Heinemann, S. H. (2006). Subtype specificity of scorpion beta-toxin Tz1 interaction with voltage-gated sodium channels is determined by the pore loop of domain 3. Mol. Pharmacol. 70, 340-347. doi: 10.1124/mol.106.024034

Lewis, R. J., Dutertre, S., Vetter, I., and Christie, M. J. (2012). Conus venom peptide pharmacology. Pharmacol. Rev. 64, 259-298. doi: 10.1124/pr.111. 005322

Li, R. A., Ennis, I. L., French, R. J., Dudley, S. C. Jr., Tomaselli, G. F., and Marbán, E. (2001). Clockwise domain arrangement of the sodium channel revealed by (mu)-conotoxin (GIIIA) docking orientation. J. Biol. Chem. 276, 11072-11077. doi: 10.1074/jbc.M010862200

Lipkind, G. M., and Fozzard, H. A. . (2000). KcsA crystal structure as framework for a molecular model of the $\mathrm{Na}(+)$ channel pore. Biochemistry $39,8161-8170$. doi: 10.1021/bi000486w

Lipkind, G. M., and Fozzard, H. A. (1994). A structural model of the tetrodotoxin and saxitoxin binding site of the Na+ channel. Biophys. J. 66, 1-13. doi: 10.1016/ S0006-3495(94)80746-5

Mannikko, R., Shenkarev, Z. O., Thor, M. G., Berkut, A. A., Myshkin, M. Y., Paramonov, A. S., et al. (2018). Spider toxin inhibits gating pore currents underlying periodic paralysis. Proc. Natl. Acad. Sci. U.S.A. 115, 4495-4500. doi: 10.1073/pnas.1720185115

Marzian, S., Stansfeld, P. J., Rapedius, M., Rinne, S., Nematian-Ardestani, E., Abbruzzese, J. L., et al. (2013). Side pockets provide the basis for a new mechanism of Kv channel-specific inhibition. Nat. Chem. Biol. 9, 507-513. doi: $10.1038 /$ nchembio. 1271

McArthur, J. R., Ostroumov, V., Al-Sabi, A., McMaster, D., and French, R. J. (2011a). Multiple, distributed interactions of mu-conotoxin PIIIA associated with broad targeting among voltage-gated sodium channels. Biochemistry 50, 116-124. doi: 10.1021/bi101316y

McArthur, J. R., Singh, G., O’Mara, M. L., McMaster, D., Ostroumov, V., Tieleman, D. P., et al. (2011b). Orientation of mu-conotoxin PIIIA in a sodium channel vestibule, based on voltage dependence of its binding. Mol. Pharmacol. 80, 219-227. doi: 10.1124/mol.111.071779 
McIntosh, J. M., Hasson, A., Spira, M. E., Gray, W. R., Li, W., Marsh, M., et al. (1995). A new family of conotoxins that blocks voltage-gated sodium channels. J. Biol. Chem. 270, 16796-16802. doi: 10.1074/jbc.270.28.16796

Minassian, N. A., Gibbs, A., Shih, A. Y., Liu, Y., Neff, R. A., Sutton, S. W., et al. (2014). Analysis of the structural and molecular basis of voltagesensitive sodium channel inhibition by the spider toxin huwentoxin-IV (mu-TRTX-Hh2a). J. Biol. Chem. 288, 22707-22720. doi: 10.1074/jbc.M113. 461392

Moczydlowski, E. G. (2013). The molecular mystique of tetrodotoxin. Toxicon 63, 165-183. doi: 10.1016/j.toxicon.2012.11.026

Narahashi, T., Deguchi, T., Urakawa, N., and Ohkubo, Y. (1960). Stabilization and rectification of muscle fiber membrane by tetrodotoxin. Am. J. Physiol. 198, 934-938. doi: 10.1152/ajplegacy.1960.198.5.934

Naylor, C. E., Bagneris, C., DeCaen, P. G., Sula, A., Scaglione, A., Clapham, D. E., et al. (2016). Molecular basis of ion permeability in a voltage-gated sodium channel. EMBO J. 35, 820-830. doi: 10.15252/embj.201593285

Noda, M., and Numa, S. (1987). Structure and function of sodium channel. J. Recept. Res. 7, 467-497. doi: 10.3109/107998987090 54998

Numa, S., and Noda, M. (1986). Molecular structure of sodium channels. Ann. N. Y. Acad. Sci. 479, 338-355. doi: 10.1111/j.1749-6632.1986.tb15580.x

Payandeh, J., Scheuer, T., Zheng, N., and Catterall, W. A. (2011). The crystal structure of a voltage-gated sodium channel. Nature 475, 353-358. doi: 10.1038/ nature 10238

Penzotti, J. L., Fozzard, H. A., Lipkind, G. M., and Dudley, S. C., and Jr. (1998). Differences in saxitoxin and tetrodotoxin binding revealed by mutagenesis of the $\mathrm{Na}+$ channel outer vestibule. Biophys. J. 75, 2647-2657. doi: 10.1016/S00063495(98)77710-0

Qu, Y., Rogers, J., Tanada, T., Scheuer, T., and Catterall, W. A. (1995). Molecular determinants of drug access to the receptor site for antiarrhythmic drugs in the cardiac Na+ channel. Proc. Natl. Acad. Sci. U.S.A. 92, 11839-11843. doi: 10.1073/pnas.92.25.11839

Sanders, S. J., Campbell, A. J., Cottrell, J. R., Moller, R. S., Wagner, F. F., Auldridge, A. L., et al. (2018). Progress in Understanding and Treating SCN2AMediated Disorders. Trends Neurosci. 41, 442-456. doi: 10.1016/j.tins.2018. 03.011

Santarelli, V. P., Eastwood, A. L., Dougherty, D. A., Horn, R., and Ahern, C. A. (2007). A cation-pi interaction discriminates among sodium channels that are either sensitive or resistant to tetrodotoxin block. J. Biol. Chem. 282, 8044-8051. doi: 10.1074/jbc.M611334200

Schantz, E. J., Ghazarossian, V. E., Schnoes, H. K., Strong, F. M., Springer, J. P., Pezzanite, J. O., et al. (1975). Structure of saxitoxin. J. Am. Chem. Soc. 97, 1238-1239. doi: 10.1021/ja00838a045

Shen, H., Zhou, Q., Pan, X., Li, Z., Wu, J., and Yan, N. (2017). Structure of a eukaryotic voltage-gated sodium channel at near-atomic resolution. Science 355:eaal4326.

Stevens, M., Peigneur, S., Dyubankova, N., Lescrinier, E., Herdewijn, P., and Tytgat, J. (2012). Design of bioactive peptides from naturally occurring muconotoxin structures. J. Biol. Chem. 287, 31382-31392. doi: 10.1074/jbc.M112. 375733

Sunami, A., Glaaser, I. W., and Fozzard, H. A. (2001). Structural and gating changes of the sodium channel induced by mutation of a residue in the upper third of IVS6, creating an external access path for local anesthetics. Mol. Pharmacol. 59, 684-691. doi: 10.1124/mol.59.4.684

Terlau, H., Heinemann, S. H., Stuhmer, W., Pusch, M., Conti, F., Imoto, K., et al. (1991). Mapping the site of block by tetrodotoxin and saxitoxin of sodium channel II. FEBS Lett. 293, 93-96. doi: 10.1016/0014-5793(91) 81159-6

Thottumkara, A. P., Parsons, W. H., and Du Bois, J. (2014). Saxitoxin. Angew. Chem. Int. Ed. Engl. 53, 5760-5784. doi: 10.1002/anie.20130 8235

Tikhonov, D. B., Bruhova, I., and Zhorov, B. S. (2006). Atomic determinants of state-dependent block of sodium channels by charged local anesthetics and benzocaine. FEBS Lett. 580, 6027-6032. doi: 10.1016/j.febslet.2006. 10.035

Tikhonov, D. B., and Zhorov, B. S. . (2012). Architecture and pore block of eukaryotic voltage-gated sodium channels in view of NavAb bacterial sodium channel structure. Mol. Pharmacol. 82, 97-104. doi: 10.1124/mol.112.078212
Tikhonov, D. B., and Zhorov, B. S. (2005a). Modeling P-loops domain of sodium channel: homology with potassium channels and interaction with ligands. Biophys. J. 88, 184-197. doi: 10.1529/biophysj.104. 048173

Tikhonov, D. B., and Zhorov, B. S. (2005b). Sodium channel activators: model of binding inside the pore and a possible mechanism of action. FEBS Lett. 579, 4207-4212. doi: 10.1016/j.febslet.2005. 07.017

Tikhonov, D. B., and Zhorov, B. S. (2017a). Conservation and variability of the pore-lining helices in P-loop channels. Channels 11, 660-672. doi: 10.1080/ 19336950.2017.1395536

Tikhonov, D. B., and Zhorov, B. S. (2017b). Mechanism of sodium channel block by local anesthetics, antiarrhythmics, and anticonvulsants. J. Gen. Physiol. 149, 465-481. doi: 10.1085/jgp.201611668

Ulbricht, W. (2005). Sodium channel inactivation: molecular determinants and modulation. Physiol. Rev. 85, 1271-1301. doi: 10.1152/physrev.00024. 2004

Van Der Haegen, A., Peigneur, S., and Tytgat, J. (2011). Importance of position 8 in mu-conotoxin KIIIA for voltage-gated sodium channel selectivity. FEBS J. 278, 3408-3418. doi: 10.1111/j.1742-4658.2011.08264.x

Vilin, Y. Y., and Ruben, P. C. (2001). Slow inactivation in voltagegated sodium channels: molecular substrates and contributions to channelopathies. Cell Biochem. Biophys. 35, 171-190. doi: 10.1385/CBB:35: 2:171

Walker, J. R., Novick, P. A., Parsons, W. H., McGregor, M., Zablocki, J., Pande, V. S., et al. (2012). Marked difference in saxitoxin and tetrodotoxin affinity for the human nociceptive voltage-gated sodium channel (Nav1.7) [corrected]. Proc. Natl. Acad. Sci. U.S.A. 109, 18102-18107. doi: 10.1073/pnas.120695 2109

Wang, J., Yarov-Yarovoy, V., Kahn, R., Gordon, D., Gurevitz, M., Scheuer, T., et al. (2011). Mapping the receptor site for alpha-scorpion toxins on a $\mathrm{Na}+$ channel voltage sensor. Proc. Natl. Acad. Sci. U.S.A. 108, 15426-15431. doi: 10.1073/pnas.1112320108

Wang, S. Y., Mitchell, J., Tikhonov, D. B., Zhorov, B. S., and Wang, G. K. (2006). How batrachotoxin modifies the sodium channel permeation pathway: computer modeling and site-directed mutagenesis. Mol. Pharmacol. 69, 788-795.

Wilson, M. J., Yoshikami, D., Azam, L., Gajewiak, J., Olivera, B. M., Bulaj, G., et al. (2011). mu-Conotoxins that differentially block sodium channels NaV1.1 through 1.8 identify those responsible for action potentials in sciatic nerve. Proc. Natl. Acad. Sci. U.S.A. 108, 10302-10307. doi: 10.1073/pnas.11070 27108

Woodward, R. B., and Gougoutas, J. Z. (1964). The structure of tetrodotoxin. J. Am. Chem. Soc. 86:5030. doi: 10.1021/ja01076a076

Xiao, Y., Blumenthal, K., and Cummins, T. R. (2014). Gating-pore currents demonstrate selective and specific modulation of individual sodium channel voltage-sensors by biological toxins. Mol. Pharmacol. 86, 159-167. doi: 10.1124/ mol.114.092338

Xue, T., Ennis, I. L., Sato, K., French, R. J., and Li, R. A. (2003). Novel interactions identified between micro -Conotoxin and the $\mathrm{Na}+$ channel domain I P-loop: implications for toxin-pore binding geometry. Biophys. J. 85, 2299-2310. doi: 10.1016/S0006-3495(03)74654-2

Yamagishi, T., Li, R. A., Hsu, K., Marban, E., and Tomaselli, G. F. (2001). Molecular architecture of the voltage-dependent $\mathrm{Na}$ channel: functional evidence for alpha helices in the pore. J. Gen. Physiol. 118, 171-182. doi: 10.1085/jgp.118. 2.171

Yan, Z., Zhou, Q., Wang, L., Wu, J., Zhao, Y., Huang, G., et al. (2017). Structure of the Nav1.4-beta1 complex from electric eel. Cell 170:470-482. doi: 10.1016/j. cell.2017.06.039

Zhang, J. Z., Yarov-Yarovoy, V., Scheuer, T., Karbat, I., Cohen, L., Gordon, D., et al. (2011). Structure-function map of the receptor site for beta-scorpion toxins in domain II of voltage-gated sodium channels. J. Biol. Chem. 286, 33641-33651. doi: 10.1074/jbc.M111.282509

Zhang, J. Z., Yarov-Yarovoy, V., Scheuer, T., Karbat, I., Cohen, L., Gordon, D., et al. (2012). Mapping the interaction site for a betascorpion toxin in the pore module of domain III of voltage-gated $\mathrm{Na}(+)$ channels. J. Biol. Chem. 287, 30719-30728. doi: 10.1074/jbc.M112. 370742 
Zhang, X., Ren, W., DeCaen, P., Yan, C., Tao, X., Tang, L., et al. (2012). Crystal structure of an orthologue of the $\mathrm{NaChBac}$ voltage-gated sodium channel. Nature 486, 130-134. doi: 10.1038/nature11054

Zhang, M. M., McArthur, J. R., Azam, L., Bulaj, G., Olivera, B. M., French, R. J., et al. (2009). Synergistic and antagonistic interactions between tetrodotoxin and mu-conotoxin in blocking voltage-gated sodium channels. Channels 3, 32-38. doi: $10.4161 /$ chan.3.1.7500

Zimin, P. I., Garic, B., Bodendiek, S. B., Mahieux, C., Wulff, H., and Zhorov, B. S. (2010). Potassium channel block by a tripartite complex of two cationophilic ligands and a potassium ion. Mol. Pharmacol. 78, 588-599. doi: 10.1124/mol. 110.064014
Conflict of Interest Statement: The authors declare that the research was conducted in the absence of any commercial or financial relationships that could be construed as a potential conflict of interest.

Copyright (c) 2018 Tikhonov and Zhorov. This is an open-access article distributed under the terms of the Creative Commons Attribution License (CC BY). The use, distribution or reproduction in other forums is permitted, provided the original author(s) and the copyright owner(s) are credited and that the original publication in this journal is cited, in accordance with accepted academic practice. No use, distribution or reproduction is permitted which does not comply with these terms. 